\title{
Novel SAHA-bendamustine hybrid NL-101 in combination with daunorubicin synergistically suppresses acute myeloid leukemia
}

\author{
JINGRUI JIN ${ }^{1,2}$, XIA LI $^{1,2}$, WENJIAN GUO ${ }^{3}$, FENGLIN LI ${ }^{1,2}$, JIANSONG HUANG $^{1,2}$, XIN HUANG $^{1,2}$, \\ JIAJIA PAN ${ }^{1,2}$, SHUJUAN HUANG ${ }^{1,2}$, WENLE YE ${ }^{1,2}$, QING LING ${ }^{1,2}$ and JIE JIN ${ }^{1,2}$ \\ ${ }^{1}$ Department of Hematology, The First Affiliated Hospital, Zhejiang University College of Medicine, Hangzhou, \\ Zhejiang 310003; ${ }^{2}$ Key Laboratory of Hematopoietic Malignancies, Diagnosis and Treatment, The First Affiliated Hospital, \\ Zhejiang University College of Medicine, Hangzhou, Zhejiang 310060; ${ }^{3}$ Department of Hematology, \\ The Second Affiliated Hospital and Yuying Children's Hospital of Wenzhou Medical University, \\ Wenzhou, Zhejiang 325027, P.R. China
}

Received May 9, 2019; Accepted February 28, 2020

DOI: 10.3892/or.2020.7591

\begin{abstract}
Acute myeloid leukemia (AML) is a highly aggressive disease with high mortality and recurrence rates, for which novel therapeutic approaches are required. Hybrid anticancer agents with dual effects have been reported to possess therapeutic potential to treat AML. However, the efficacy and underlying toxicity of these hybrids in combination with other agents remain unclear. NL-101 is a novel hybrid formed by fusing the DNA damage-inducing agent bendamustine with the histone deacetylase inhibitor vorinostat. In the present study, NL-101 treatment was combined with the conventional chemotherapeutic drug daunorubicin (DNR) in AML cells, and it was revealed that these two compounds exerted synergistic anti-AML effects. In addition, NL-101 enhanced DNR-induced cell apoptosis, as assessed by flow cytometry and as indicated by the upregulation of cleaved-poly (ADP-ribose) polymerase, cleaved-caspase-3, cleaved-caspase-7, BAD and BIM. Mechanistically, the DNA double-strand breaks marker $\gamma-\mathrm{H} 2 \mathrm{AX}$, and other proteins associated with DNA damage, were investigated, and it was demonstrated that NL-101 in combination with DNR synergistically promoted the DNA damage response. In vivo, this combination significantly delayed the progression of AML and prolonged the survival time in mice. Collectively, the present results suggested that
\end{abstract}

Correspondence to: Professor Jie Jin, Department of Hematology, The First Affiliated Hospital, Zhejiang University College of Medicine, 79 Qingchun Road, Shangcheng, Hangzhou, Zhejiang 310003, P.R. China

E-mail: jiej0503@zju.edu.cn

Abbreviations: AML, acute myeloid leukemia; SAHA, vorinostat; DNR, daunorubicin; Ara-C, cytarabine; CI, combination index; DSBs, DNA double-strand breaks; DMSO, dimethyl sulfoxide; FITC, fluorescein isothiocyanate; PI, propidium iodide; B-NSG, NOD-Prkdc ${ }^{\text {scid }}$ IL $2 \mathrm{rg}^{\text {tm1 } 1} /$ Bcgen

Key words: AML, NL-101, DNR, apoptosis, DNA damage
NL-101 in combination with daunorubicin could be an alternative novel therapeutic strategy for treating leukemia.

\section{Introduction}

Acute myeloid leukemia (AML) is a heterogeneous malignant hematopoietic stem cell disease, which is commonly characterized by an abnormal accumulation of myeloblasts (1). Chemotherapy remains the most common treatment for AML (2). The current standard clinical approach is known as ' $3+7$ induction', and consists of a combination of daunorubicin (DNR), at a dosage of $60-90 \mathrm{mg} / \mathrm{m}^{2}$ for 3 days, and cytarabine (Ara-C), at a dosage of $100-200 \mathrm{mg} / \mathrm{m}^{2}$ for 7 days $(3,4)$. Although most patients with AML exhibit a response to induction chemotherapy, relapse represents the main cause of treatment failure (5). Patients with secondary AML have a 5-year survival rate of 5-10\% (6,7). Moreover, it has previously been demonstrated that high-dose chemotherapy is not safe for treating elderly patients ( $>60$ years old) due to poor drug tolerance and high toxicity $(8,9)$. The overall prognosis of AML remains unsatisfactory $(10,11)$; therefore, the development of safe and effective therapy for AML is necessary to improve the prognosis of patients with this malignancy.

Due to advances in disease and drug research, numerous novel treatments have been developed to treat and manage AML $(12,13)$; however, few novel drugs have been approved for the treatment of AML in the past 20 years (14). The effectiveness of some complexes, including CPX-351 (15-17), liposome-encapsulated DNR and Ara-C, prompted the identification of a more potent and effective hybrid. NL-101 (also known as EDO-S101) is an alkylate, and is a novel chemotherapeutic agent structurally consisting of the DNA damage-inducing agent bendamustine hydrochloride and the histone deacetylase inhibitor vorinostat (SAHA) (18). NL-101 was designed to allow the simultaneous function of the two drugs and has been reported to have an increased efficacy compared with the single agents (19). It has previously been shown that NL-101 upregulated the protein expression levels of cleaved-poly (ADP-ribose) polymerase (PARP) and cleaved-caspase-3 in Kasumi and NB4 cells. In addition, 
NL-101 could increase the survival rate in mice compared with bendamustine and SAHA in vivo (20). However, the application of NL-101 with other drugs needs to be further investigated.

Anthracyclines are one of the most effective anticancer drugs developed (21). DNR is an anthracycline that has been broadly used for the treatment of cancer (22). The mechanism underlying DNR-mediated cytotoxicity has been reported to include the induction of DNA damage, causing DNA double-strand breaks (DSBs) and apoptosis $(23,24)$. The hybrid NL-101 in combination with DNR may represent a novel strategy to improve the therapeutic effects of NL-101, thus facilitating the development of more effective combinatorial therapies to treat AML.

The present study aimed to investigate the potential of the novel hybrid NL-101 to treat AML and to examine its synergistic effect in combination with DNR. In addition, the mechanisms underlying the effect of NL-101 in combination with DNR were examined in vitro, and cell-mediated xenograft AML models were used to investigate the efficacy and safety of this combinatorial regimen in vivo.

\section{Materials and methods}

Patients. Clinical data were collected from the medical records of patients with AML at Key Laboratory of Hematopoietic Malignancies, Diagnosis and Treatment, The First Affiliated Hospital, Zhejiang University College of Medicine, (Hangzhou, China). From March 2017 to July 2018, 10 patients were included in this study with demographic and clinical information (Table SI), including diagnosis, age, French-American-British classification system type (25), cytogenetics and molecular mutation. Patients with secondary AML or acute promyelocytic leukemia were excluded. This study was approved by the Research Ethics Committee of the First Affiliated Hospital, College of Medicine, Zhejiang University (Hangzhou, China). Informed consent was obtained from each patient according to institutional guidelines.

Antibodies and reagents. Antibodies against caspase-3 (cat. no. 9662), caspase-7 (cat. no. 9492), PARP (cat. no. 9532), BAD (cat. no. 9239), BIM (cat. no. 2933), cyclin B1 (cat. no. 4135), cell division cycle protein 2 (CDC2; cat. no. 9116), $\gamma$-H2AX (Ser139; cat. no. 9718), phosphorylated (p)-ATR (Thr1981; cat. no. 30632), p-ATM (Ser1981; cat. no. 5883), p-CHK1 (Ser345; cat. no. 2348), p-CHK2 (Thr68; cat. no. 2197), GAPDH (cat. no. 5174) and $\beta$-actin (cat. no. 4970) were purchased from Cell Signaling Technology, Inc. ATR (cat. no. 19787-1-AP), ATM (cat. no. 27156-1-AP), CHK1 (cat. no. 10362-1-AP) and CHK2 (cat. no. 13954-1-AP) antibodies were purchased from ProteinTech Group, Inc. Anti-human (h) CD45-fluorescein isothiocyanate (FITC) (cat. no. ab134199) was obtained from Abcam. DNR was purchased from Selleck Chemicals. NL-101 was provided from Minsheng Institute of Pharmaceutical Research. DNR was diluted to $1-100 \mu \mathrm{M}$ with PBS, whereas NL-101 was diluted to $100-1,000 \mu \mathrm{M}$ with dimethyl sulfoxide (DMSO). Aliquots of DNR and NL-101 were stored at $-20^{\circ} \mathrm{C}$. Female NOD-Prkdc ${ }^{\text {scid }}$ IL $2 \mathrm{rg}^{\mathrm{tm} 1} / \mathrm{Bcgen}$ (B-NSG) mice (age, 6-8 weeks; weight, 18-22 g; Biocytogen) were used to generate a xenograft model of AML.
Cell lines and primary cells. AML cell lines MV4-11, Molm13 and THP-1 were provided by Professor Ravi Bhatia (City of Hope National Medical Center, Duarte, CA, USA). HL-60 and Kasumi-1 cell lines were purchased from American Type Culture Collection. NOMO-1, OCI-AML-2 and OCI-AML-3 cell lines were gifted by Professor Jianjun Chen (City of Hope, Gehr Family Center for Leukemia Research, Duarte, CA, USA). The MV4-11-luciferase (luc) cell line was gifted by Professor Rongzhen $\mathrm{Xu}$ (The Second Affiliated Hospital of Zhejiang University, Hangzhou, China). MV4-11 and Molm13 cells were cultured in Iscove's Modified Dulbecco's Medium (Gibco; Thermo Fisher Scientific, Inc.) and the other cells were cultured in RPMI-1640 medium (Gibco; Thermo Fisher Scientific, Inc.) supplemented with $10 \%$ fetal bovine serum (Gibco; Thermo Fisher Scientific, Inc.) at $37^{\circ} \mathrm{C}$ in a humidified incubator containing $5 \% \mathrm{CO}_{2}$. Peripheral blood mononuclear cells were isolated by Ficoll-Hypaque (Sigma-Aldrich; Merck KGaA) density gradient centrifugation after obtaining written informed consent from patients with AML. All procedures were conducted in accordance with the ethical standards of the Research Ethics Committee of the First Affiliated Hospital, Zhejiang University College of Medicine.

Cell viability assay. This assay is also known as the cell proliferation assay $(26,27)$. AML cells $\left(2 \times 10^{5}\right)$ or primary AML cells ( $1 \times 10^{6}$; PBMCs isolated from patients with AML) were plated into 96-well plates. After treatment with NL-101 $(150,300,450,600$ and $750 \mathrm{nM}$ in MV4-11; 250, 500, 750, 1,000 and 1,250 nM in HL-60; 100, 200, 400 and $800 \mathrm{nM}$ in primary AML cells), DNR $(4,8,12,16$ and $20 \mathrm{nM}$ in MV4-11; 8, 16, 24, 32 and $40 \mathrm{nM}$ in HL-60; 4, 8, 16 and $32 \mathrm{nM}$ in primary AML cells), the combination or DMSO at $37^{\circ} \mathrm{C}$ for $48 \mathrm{~h}, 20 \mu \mathrm{l}$ MTS solution (Promega Corporation) was added. Subsequently, the plates were read at an absorbance of $490 \mathrm{~nm}$ after $3-4 \mathrm{~h}$ incubation at $37^{\circ} \mathrm{C}$. Experiments were repeated three times.

Flow cytometric analysis. For assessment of apoptosis, cells $\left(2 \times 10^{5} / \mathrm{ml}\right)$ were treated with corresponding drugs for $48 \mathrm{~h}$ and then collected. Cells were resuspended in $1 \mathrm{X}$ binding buffer, then co-stained with $5 \mu \mathrm{l}$ Annexin V-FITC and $10 \mu \mathrm{l}$ propidium iodide (PI), obtained from an apoptosis detection kit (Multi Sciences), for $15 \mathrm{~min}$ at room temperature in the dark. To analyze cell cycle distribution, cells were treated with drugs for $24 \mathrm{~h}$. After fixing with $75 \%$ ethanol at $4^{\circ} \mathrm{C}$ overnight, the cells were resuspended in buffer with $50 \mu \mathrm{g} / \mathrm{ml}$ PI and $100 \mu \mathrm{g} / \mathrm{ml}$ RNase A, obtained from a cell cycle detection kit (Multi Sciences), for $30 \mathrm{~min}$ at room temperature. To assess hCD $45^{+}$cells, the bone marrows of AML mice were incubated with 1:25 anti-hCD45 as aforementioned. The engrafted MV4-11 cells were analyzed using anti-hCD45 FITC-labeled antibody (Abcam). The apoptotic cells, DNA content and hCD45 ${ }^{+}$cells were analyzed using NovoExpress software (version 1.2.1; ACEA Biosciences, Inc.) and FACScan flow cytometer (BD Biosciences).

Western blot analysis. Cells $\left(2 \times 10^{5} / \mathrm{ml}\right)$ were treated with NL-101, DNR, NL-101 + DNR or DMSO for 24 or $48 \mathrm{~h}$ at $37^{\circ} \mathrm{C}$, and were then collected. After lysing in 1X RIPA buffer (Cell Signaling Technology, Inc.) on ice for $30 \mathrm{~min}$, cells were 
centrifuged at $12,000 \mathrm{xg}$ for $15 \mathrm{~min}$ at $4^{\circ} \mathrm{C}$. The supernatant was then collected and protein concentration was determined using bicinchoninic acid reagent. Protein samples $(50 \mu \mathrm{g})$ were separated by 10-12\% SDS-PAGE (Thermo Fisher Scientific, Inc.) and transferred to PVDF membranes (EMD Millipore). Subsequently, the membranes were blocked in 5\% non-fat milk for $1-1.5 \mathrm{~h}$ at room temperature and incubated with primary antibodies $(1: 1,000)$ at $4^{\circ} \mathrm{C}$ for $\geq 4 \mathrm{~h}$. The membranes were then washed with TBS-Tween $(0.1 \%)$ three times and were incubated with 1:5,000 anti-rabbit or anti-mouse secondary antibodies (1:5,000; cat. nos. 7074 and 7076; Cell Signaling Technology, Inc.) at room temperature for 1-1.5 h. The protein bands were visualized with SuperSignal ${ }^{\text {TM }}$ West Femto Maximum Sensitivity Substrate (cat. no. 34096; Thermo Fisher Scientific, Inc.), and images were captured using a chemiluminescence imager (ChemiDoc XRS+; Bio-Rad Laboratories, Inc.) and were analyzed using Image Lab software (version 5.2.1; Bio-Rad Laboratories, Inc.).

AML mice models. All mice were raised under standard conditions (room temperature, $22-24^{\circ} \mathrm{C} ; 12$-h light/dark cycle; relative humidity, 45-50\%), with free access to food and water. For the MV4-11-luc model, 1×106 MV4-11-luc cells were implanted intravenously into the tail vein of mice. Mice were randomly sorted into four groups ( $n=5 /$ group), and were observed daily and weighed every 3 days. The leukemic burden was estimated by non-invasive luciferin imaging (28) every 7 days. Mice were treated with $12 \mathrm{mg} / \mathrm{kg} \mathrm{NL}-101,1.5 \mathrm{mg} / \mathrm{kg}$ DNR, the combination or the same volume of PBS (vehicle). NL-101 was given intravenously on days 1 and 2, and DNR was given intravenously on days 1,3 , and 5 after AML symptoms were observed (after 7 days). Mice were observed daily and hind limb paralysis was used as an endpoint (28). When lower extremities appeared paralyzed, the mice were euthanized in a non-pre-inflated chamber. The chamber displacement rate of $\mathrm{CO}_{2}$ was set at $20 \%$ per min. Euthanasia was confirmed by cervical dislocation. All mice were euthanized before the study finished. Subsequently, the bone marrows obtained from the lower extremities of AML mice were washed three times and resuspended in $250 \mu \mathrm{l}$ PBS. Anti-hCD45 antibody (10 $\mu \mathrm{l}$; equivalent to 1:25) was then added to the samples for $30 \mathrm{~min}$ at $4^{\circ} \mathrm{C}$ in the dark. The hCD $45^{+}$ cells were detected by flow cytometric analysis, as aforementioned. Animal experiments were approved by the Animal Experimental Ethical Inspection of the First Affiliated Hospital, College of Medicine, Zhejiang University.

Statistical analysis. The combination index (CI) was calculated by CalcuSyn software (version 2.1; Biosoft). GraphPad Prism software (version 6.0; GraphPad Software, Inc.) was used to calculate $\mathrm{IC}_{50}$. The experiments were repeated three times. The cell viability assay was analyzed by one-way ANOVA followed by LSD multiple comparisons test, whereas the other biological and in vivo assays were analyzed by one-way ANOVA followed by Tukey's test using SPSS version 19.0 (IBM Corp.).

\section{Results}

NL-101 in combination with DNR synergistically inhibits proliferation of AML cell lines and primary AML cells. The present study examined the inhibitory effects of the single agent NL-101 on the proliferation of AML cells. AML cells were plated into 96 -well plates and exposed to increasing concentrations of NL-101 (200, 400, 600, 800, 1,000, 1,500 and 2,000 $\mathrm{nM}$ ) and equal volumes of DMSO for $48 \mathrm{~h}$. The results revealed that NL-101 inhibited the growth of AML cell lines and primary AML cells; the $\mathrm{IC}_{50}$ values are shown in Table I. Subsequently the synergistic effects of NL-101 and DNR were assessed on two cell lines: MV4-11 and HL-60 cells. A previous study indicated that the concentration at which the inhibition rates of the two single agents were similar was the most suitable as the combined concentration to calculate the combination index (28). Thus we confirmed the concentration of NL-101 and DNR in the combination experiments. In the present study, NL-101 and DNR synergistically inhibited the growth of AML cell lines (Fig. 1A and B) and primary AML cells (Fig. 1C-E). The synergistic effect curves were generated using CalcuSyn. CIs at the 50, 75 and $90 \%$ effective dose are presented in Tables II and III. The present study demonstrated that NL-101 in combination with DNR had a synergistic inhibitory effect on the proliferation of AML cell lines and primary AML cells. The demographic information of the included patients is presented in Table SI.

Combination treatment with NL-101 and DNR induces cell apoptosis and $G_{2} / M$ cell cycle arrest. To explore the mechanism underlying the synergistic effect of NL-101 and DNR, cell apoptosis and cell cycle arrest was assessed after exposure to drugs. Cells were treated with NL-101, DNR or the combination for 48 h. Compared with untreated cells and single agent-treated cells, combination treatment with NL-101 and DNR resulted in a significant increase in apoptosis (early + late) of MV4-11 and HL-60 cells $(\mathrm{P}<0.05$; Fig. $2 \mathrm{~A}$ and $\mathrm{B})$. Subsequently, the key signaling proteins in the apoptotic pathway were analyzed by western blot analysis. As shown in Fig. 2C, the combination treatment markedly increased the expression of cleaved-PARP, cleaved-caspase- 3 and cleaved-caspase-7 after 48 h. In addition, the expression levels of BAD and BIM were increased by 48 -h treatment with NL-101 in combination with DNR (Fig. 2D). After treatment for $24 \mathrm{~h}$, cell cycle distribution was analyzed. There was no significant evidence to suggest that the combination of NL-101 and DNR synergistically impeded cell cycle progression of MV4-11 cells (Fig. S1A). However, significant $\mathrm{G}_{2} / \mathrm{M}$ accumulation was observed following treatment of HL-60 cells with the combination ( $\mathrm{P}<0.05$; Fig. S1B). Western blot analysis revealed an upregulation of the $\mathrm{G}_{2} / \mathrm{M}$ regulatory molecules, cyclin $\mathrm{B} 1$ and $\mathrm{CDC} 2$, in the combination treatment group compared with the single agent groups in HL-60 cells (Fig. S1C). These findings indicated that NL-101 in combination with DNR may have the ability to induce apoptosis and $\mathrm{G}_{2} / \mathrm{M}$ cell cycle arrest of HL- 60 cells.

NL-101 in combination with DNR increases the DNA damage response. By further investigating the cause of apoptosis, western blot analysis revealed that the protein expression levels of $\gamma$-H2AX were increased in cells treated with the drug combination compared with the single agents. In addition, the expression levels of DNA damage checkpoint proteins, p-ATM, p-CHK1 and p-CHK2, were upregulated after treatment (Fig. 3). P-ATR was upregulated in HL-60 cells, whereas 
Table $\mathrm{I}_{\text {. }} \mathrm{IC}_{50}$ values of NL-101 in AML cell lines and primary AML cells.

\begin{tabular}{lc}
\hline Cell type & $\mathrm{IC}_{50}$ value $(\mathrm{nM})$ \\
\hline AML cell lines & \\
Molm13 & $666.2 \pm 24.0$ \\
MV4-11 & $805.7 \pm 58.8$ \\
Kasumi-1 & $973.1 \pm 61.4$ \\
OCI-AML-3 & $941.4 \pm 105.9$ \\
OCI-AML-2 & $962.5 \pm 49.5$ \\
HL-60 & $1,124.0 \pm 68.4$ \\
NOMO-1 & $1,338.0 \pm 53.9$ \\
THP1 & $1,466.0 \pm 62.4$ \\
Primary AML cells & \\
AML1 & 281.1 \\
AML2 & 312.5 \\
AML3 & 324.4 \\
AML4 & 473.2 \\
AML5 & 528.2 \\
AML6 & 632.0 \\
AML7 & 661.0 \\
AML8 & 741.4 \\
AML9 & $1,017.0$ \\
AML10 & $1,034.0$ \\
\hline
\end{tabular}

AML, acute myeloid leukemia.

Table II. Synergistic effect of NL-101 in combination with DNR on MV4-11 and HL-60 cells.

\begin{tabular}{lccc}
\hline & \multicolumn{3}{c}{ CI values } \\
\cline { 2 - 4 } Cell line & $\mathrm{ED}_{50}$ & $\mathrm{ED}_{75}$ & $\mathrm{ED}_{90}$ \\
\hline MV4-11 & 0.87436 & 0.68794 & 0.55617 \\
HL-60 & 0.74289 & 0.51788 & 0.36108 \\
\hline
\end{tabular}

CI, combination index; DNR, daunorubicin; ED, effective dose.

no change was observed in MV4-11 cells. ATR, ATM, CHK1 and CHK2 exhibited no change in MV4-11 and HL-60 cells. Briefly, increased DNA damage may contribute to apoptosis of cells induced by the combination treatment.

NL-101 in combination with DNR delays AML progression and prolongs survival in vivo. This study also investigated the efficacy and safety of NL-101 in combination with DNR on female B-NSG MV4-11-luc xenograft mouse models. Mice were randomly sorted into four treatment groups. Briefly, $1 \times 10^{6}$ MV4-11-Luc cells were injected into mice; 7 days after cell injection, engraftment was measured by photon intensity and leukemia burden was assessed weekly. The treatment regimen is presented in Fig. 4A. On day 22 after transplantation, the fluorescence intensity of the combination group was
Table III. Synergistic effect of NL-101 in combination with DNR on primary AML cells.

\begin{tabular}{lccc}
\hline & \multicolumn{3}{c}{ CI values } \\
\cline { 2 - 4 } & $\mathrm{ED}_{50}$ & $\mathrm{ED}_{75}$ & $\mathrm{ED}_{90}$ \\
\hline AML4 & 0.91606 & 0.61831 & 0.46426 \\
AML5 & 0.85689 & 0.80696 & 0.89024 \\
AML9 & 0.78682 & 0.74980 & 0.74815 \\
\hline
\end{tabular}

AML, acute myeloid leukemia; CI, combination index; DNR, daunorubicin; ED, effective dose.

distinctly reduced compared with the single drug treatment group ( $\mathrm{P}<0.001$; Fig. 4B and $\mathrm{C}$ ). Blast cell number in the bone marrow was quantified and the number of hCD $45^{+}$cells was significantly decreased in the combination treatment group $(\mathrm{P}<0.001$; Fig. 4D). In addition, combination treatment significantly prolonged survival time $(\mathrm{P}<0.05$; Fig. $4 \mathrm{E})$. These data indicated that NL-101 in combination with DNR enhanced the therapeutic effect on AML, compared with untreated and single agents, in vivo.

\section{Discussion}

In the present study, the efficacy of NL-101 was investigated in AML cells, and the synergistic anti-AML effects of NL-101 in combination with DNR were revealed. Notably, the mechanism underlying NL-101 function was investigated and it was suggested that this synergistic effect was due to an increase in DNA damage-induced apoptosis. In addition, NL-101 + DNR treatment delayed AML progression and prolonged survival time in vivo.

The SAHA-bendamustine hybrid NL-101 has dual molecular mechanisms and has been reported to exhibit improved pharmaceutical effects in treating AML compared with single agents (20). In the present study, treatment with NL-101 alone inhibited proliferation of AML cell lines and primary AML cells in a dose-dependent manner. In order to identify a novel regimen that could potentially improve the prognosis of patients with AML, NL-101 treatment was combined with DNR, one of the most effective chemotherapeutic drugs used to treat patients with AML. Subsequently, the synergistic effects of this combinatorial therapy were identified in various types of AML cells, including MV4-11 and HL-60 cell lines, and primary AML cells; the synergistic effect was reflected by $\mathrm{CI}<1$. Notably, an increase in cell apoptosis may be responsible for this effect. Cleaved-PARP has been reported to serve a role in apoptosis and in numerous types of programmed cell death (29,30). In addition, caspases (31) and the Bcl-2 family proteins $(32,33)$ have been demonstrated to serve a role in apoptosis. In the present study, a significant upregulation in the protein expression levels of cleaved-PARP, cleaved-caspase-3, cleaved-caspase-7, BAD and BIM was detected in AML cells following treatment. Furthermore, $\mathrm{G}_{2} / \mathrm{M}$ cell cycle arrest was observed, and an upregulation in the $\mathrm{G}_{2} / \mathrm{M}$ regulatory molecules, cyclin B1 and CDC2, was detected in HL-60 cells. 
A

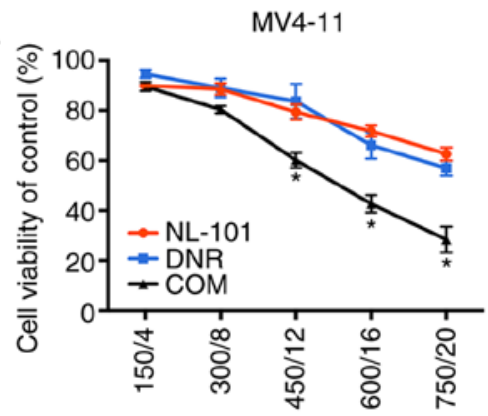

NL-101/DNR (nM/nM)

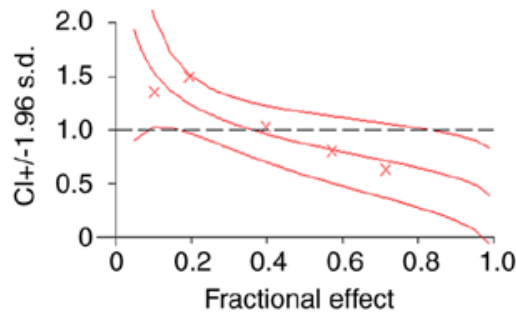

B

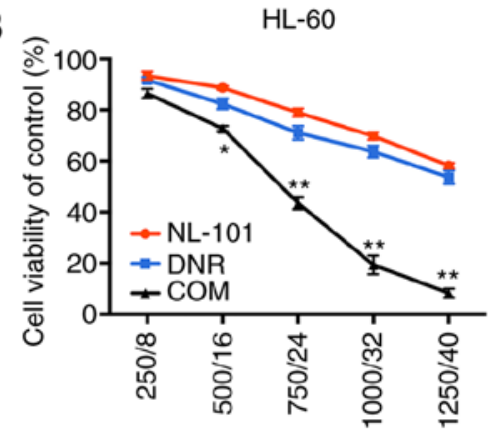

NL-101/DNR (nM/nM)

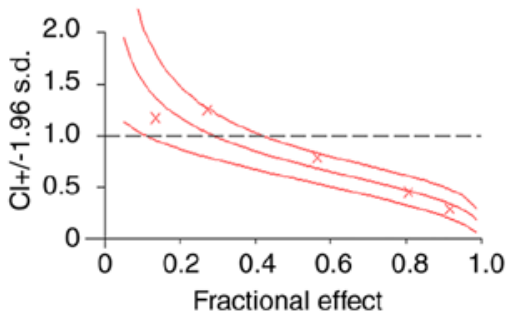

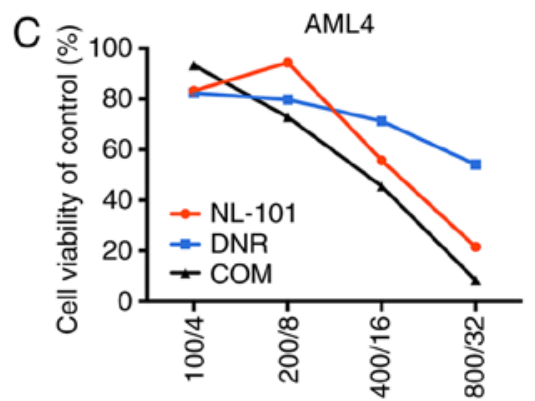

NL-101/DNR (nM/nM)

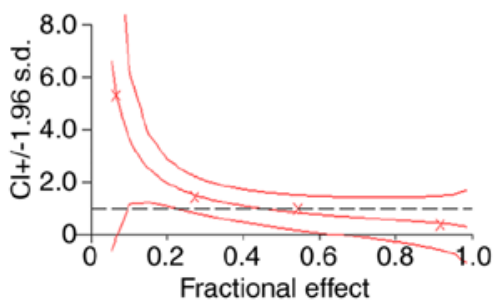

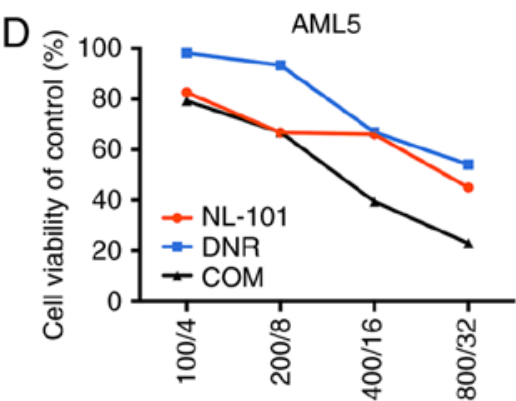

NL-101/DNR (nM/nM)

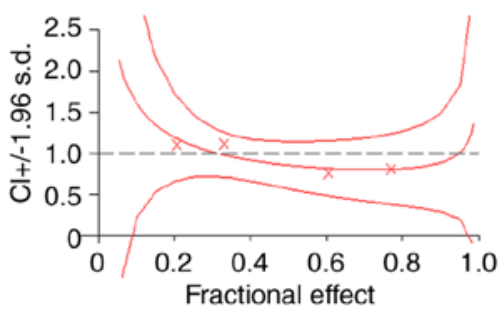

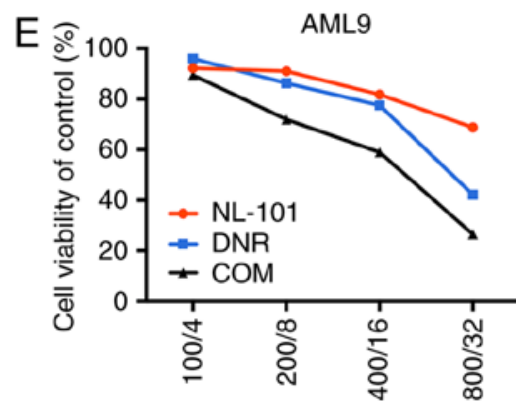

NL-101/DNR (nM/nM)

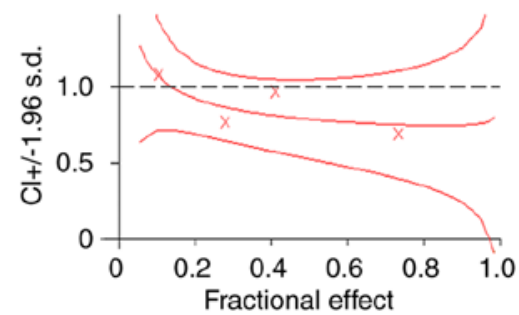

Figure 1. NL-101, DNR and a combination of these agents inhibit the growth of AML cells. (A) MV4-11 cells, (B) HL-60 cells and (C-E) primary AML cells were treated with NL-101, DNR and the combination for $48 \mathrm{~h}$. The rate of viability was measured using a MTS assay. The findings were normalized to the control group. Data are presented as mean viability rates $\pm \mathrm{SD}$ from at least three independent experiments. P-values were determined using one-way ANOVA. ${ }^{*} \mathrm{P}<0.05$ and ${ }^{* *} \mathrm{P}<0.001$ vs. NL-101 and DNR groups. AML, acute myeloid leukemia; CI, combination index; COM, combination; DNR, daunorubicin.

In some AML cells, it has been suggested that cell cycle arrest contributes to cell death; however, this process is not absolute in $\mathrm{AML}$ and requires further validation. Therefore, the present findings suggested that NL-101 in combination with DNR had the ability to synergistically inhibit AML cell proliferation by principally enhancing apoptosis.

Subsequently, the mechanisms underlying enhancement of apoptosis following combination therapy were investigated; it was revealed that $\gamma$-H2AX, p-ATM, p-CHK1 and p-CHK2 were upregulated in MV4-11 and HL-60 cells, whereas no changes in the expression levels of ATR, ATM, CHK1 and CHK2 were detected, following combination treatment. Since the bendamustine subunit in NL-101 $(34,35)$ and DNR $(36,37)$ are known to induce the DNA damage stress response, it was hypothesized that the synergistic effects of NL-101 and DNR may be caused by an enhancement of the DNA damage stress response. The protein expression of $\gamma$-H2AX is a sensitive DSB response marker $(38,39)$, and ATR, in combination with ATM, is required for the precise repair of DSBs $(40,41)$. ATR and ATM can phosphorylate and activate $\mathrm{CHK} 1$ and $\mathrm{CHK} 2$, which have important roles in the DNA damage checkpoint (42). However, in the present study, p-ATR was significantly upregulated in HL-60 cells but not in MV4-11 cells. This discrepancy may be responsible for the inconsistency in cell cycle distribution in MV4-11 and HL-60 cells. Collectively, it was suggested that, by enhancing DNA damage, NL-101 + DNR synergistically increased cell apoptosis and led to AML cell death. 
A

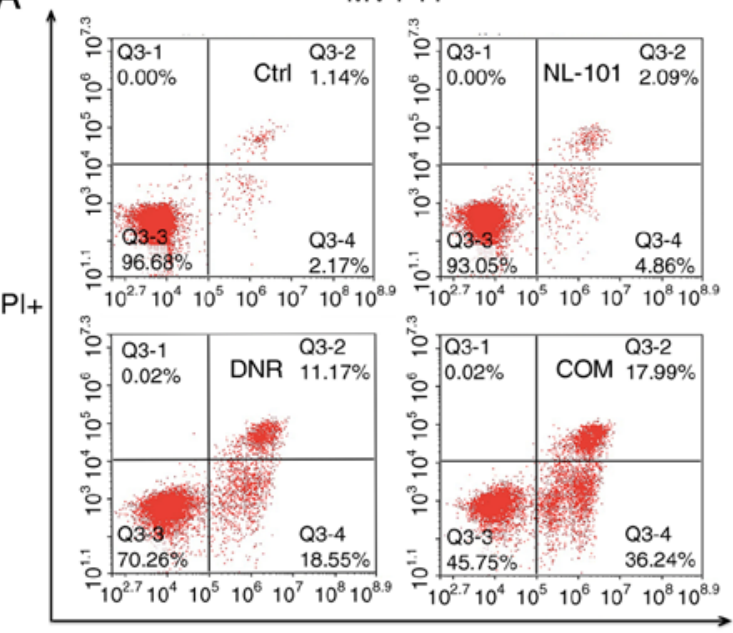

Annexin $\mathrm{V}_{+}$

$\mathrm{B}$

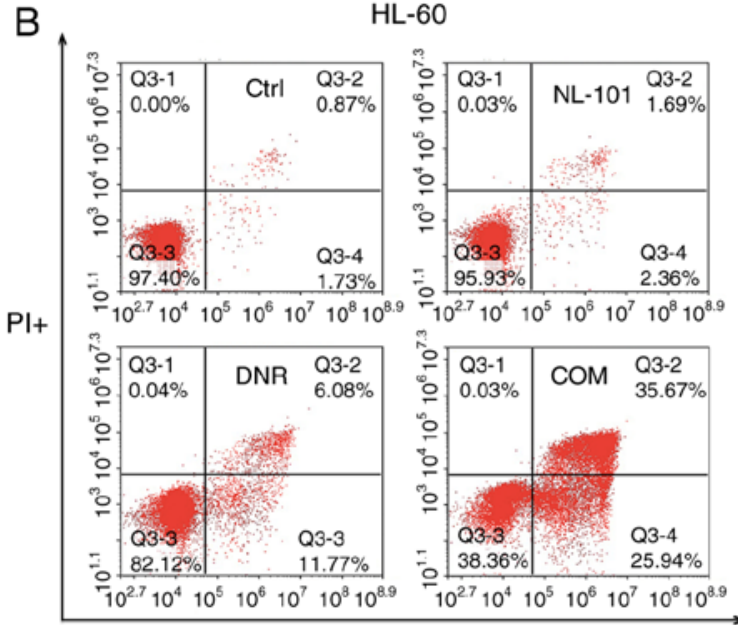

Annexin $\mathrm{V}+$
MV4-11

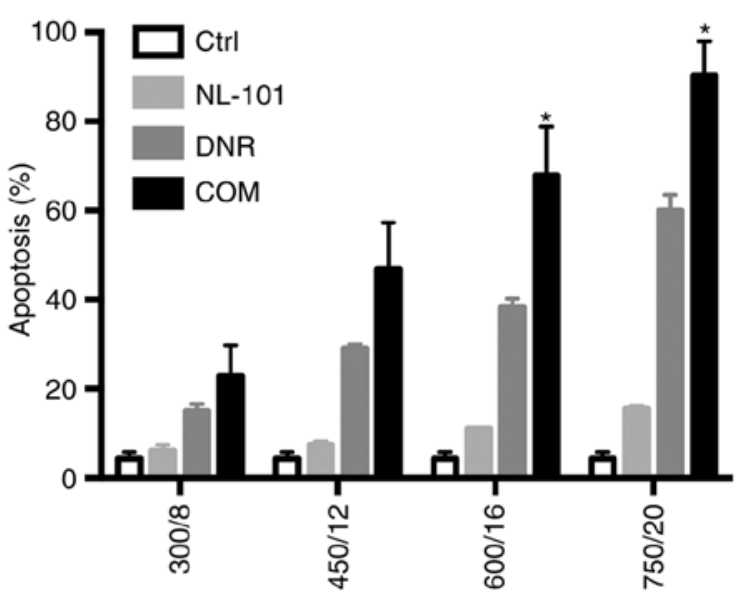

NL-101/DNR (nM/nM)

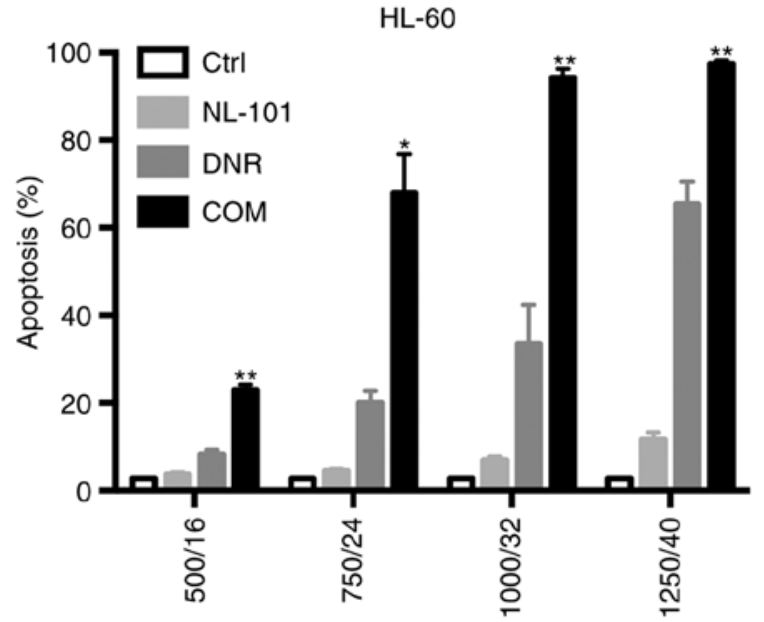

NL-101/DNR (nM/nM)

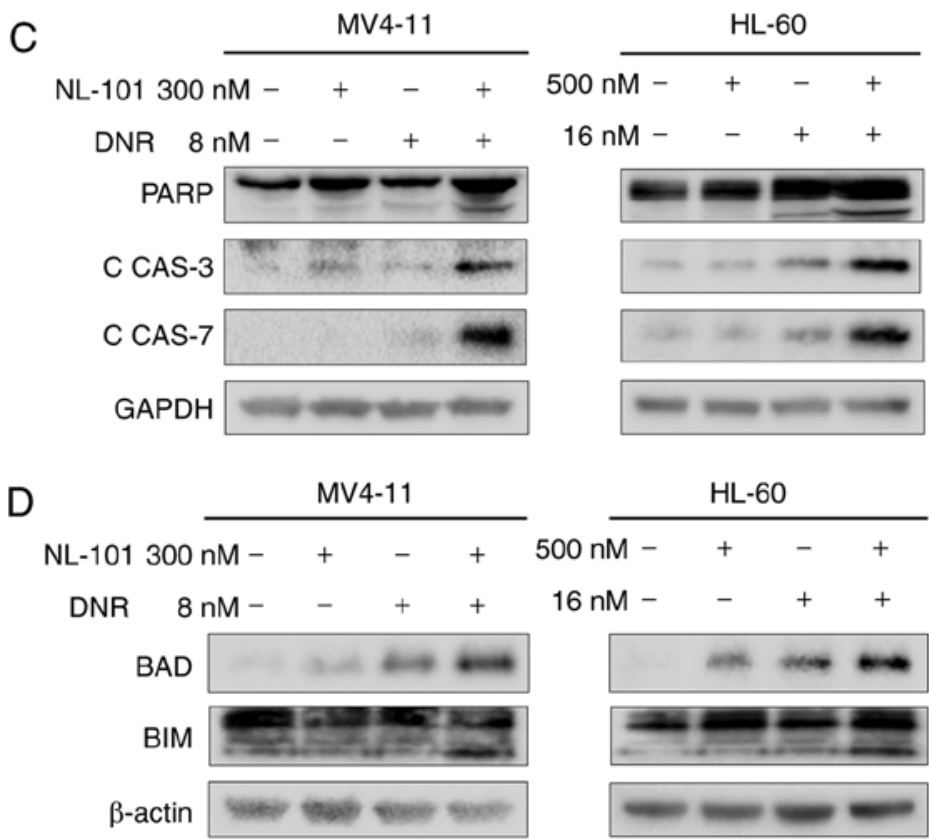

Figure 2. Apoptosis is induced by NL-101, DNR and a combination of these agents in acute myeloid leukemia cells. (A) MV4-11 and (B) HL-60 cells were treated with NL-101, DNR and the combination for $48 \mathrm{~h}$. Cells co-stained with Annexin V and PI were measured by flow cytometry. Expression of (C) cleaved-PARP, cleaved-CAS3, cleaved-CAS7, (D) BAD and BIM were detected in MV4-11 and HL-60 cells by western blotting after cells were exposed to drugs for $48 \mathrm{~h}$. Data are presented as the mean apoptosis rates $\pm \mathrm{SD}$. P-values were determined using one-way ANOVA. ${ }^{*}<0.05$ and ${ }^{* *} \mathrm{P}<0.001$ vs. NL-101 and DNR groups. C, cleaved; CAS, caspase; COM, combination; Ctrl, control; DNR, daunorubicin; PARP, poly (ADP-ribose) polymerase; PI, propidium iodide. 

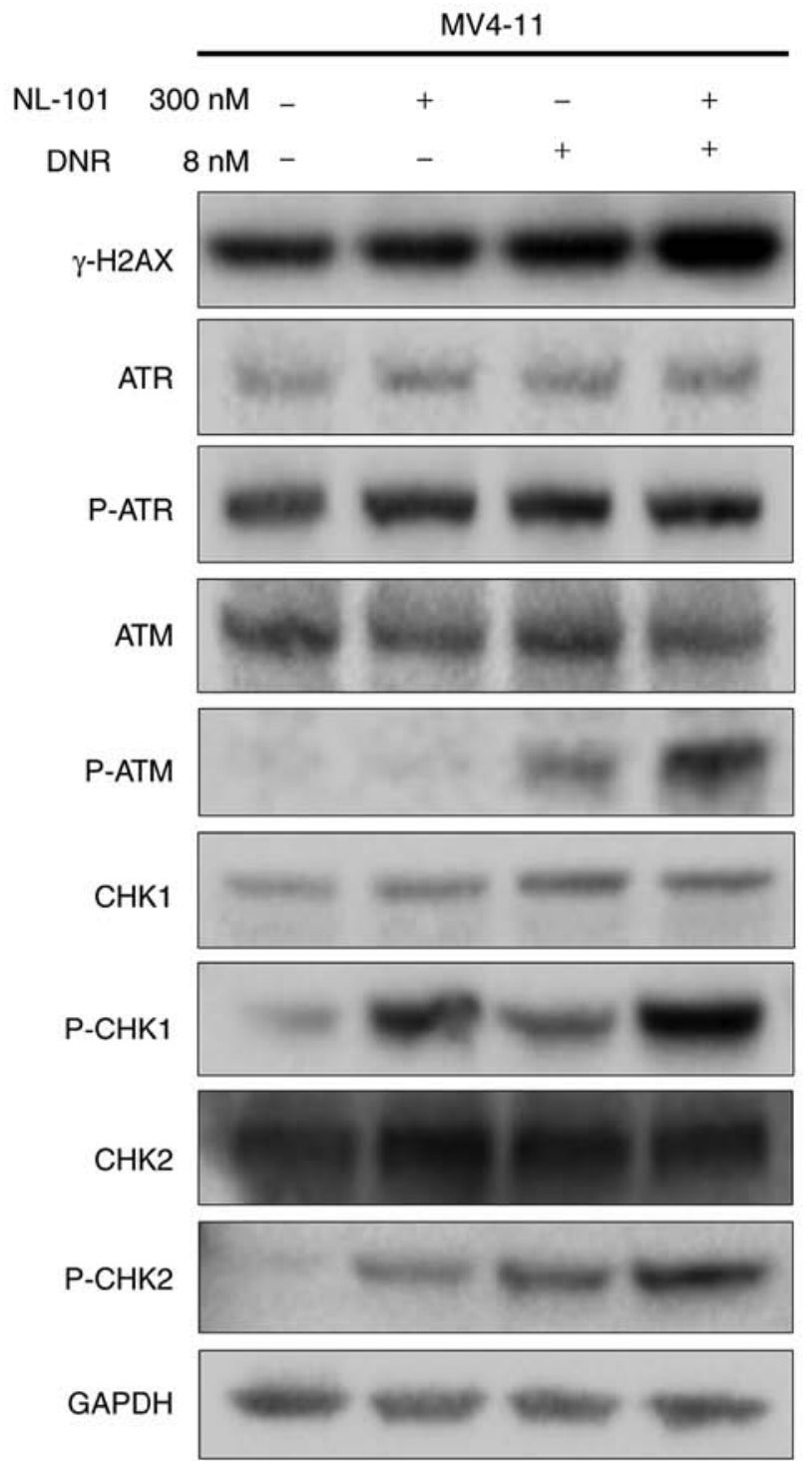

\begin{tabular}{rrrrr}
\multicolumn{7}{c}{$\mathrm{HL}-60$} \\
\hline $500 \mathrm{nM}$ & - & + & - & + \\
$16 \mathrm{nM}$ & - & - & + & +
\end{tabular}
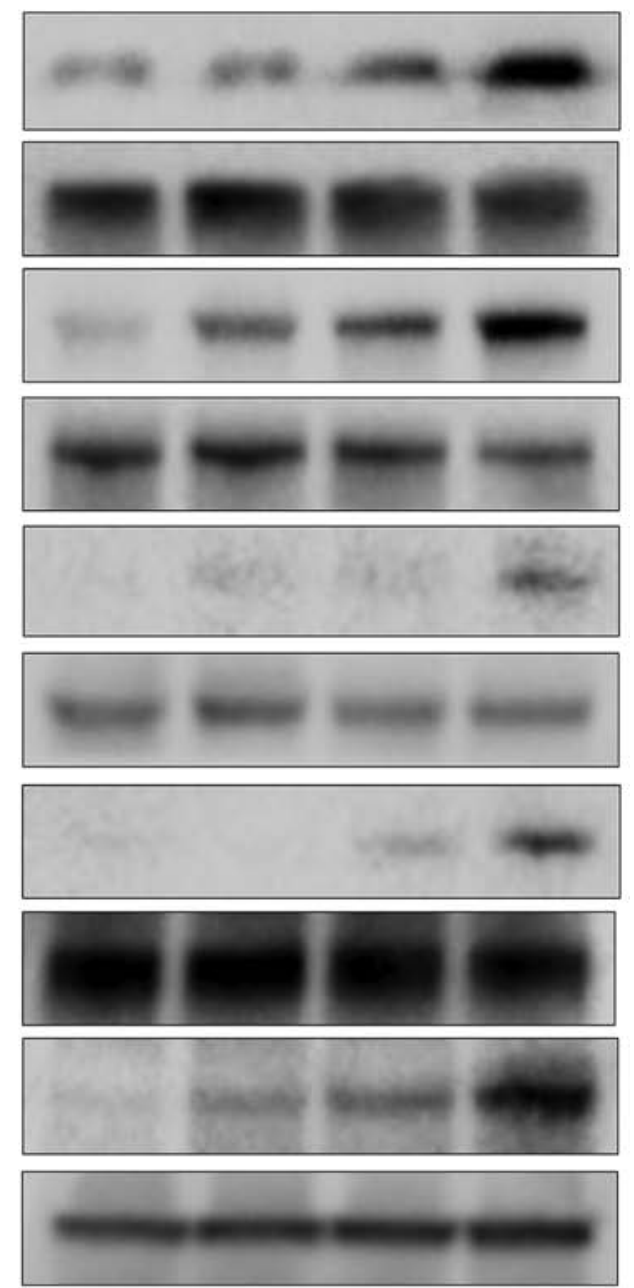

Figure 3. NL-101 in combination with DNR promotes DNA damage-related signaling. MV4-11 cells were treated with 300 nM NL-101, 8 nM DNR and the combination; HL-60 cells were treated with $500 \mathrm{nM} \mathrm{NL}-101,16 \mathrm{nM}$ DNR and the combination for $48 \mathrm{~h}$. Western blot analysis was conducted for $\gamma$-H2AX, ATR, p-ATR, ATM, p-ATM, CHK1, p-CHK1, CHK2 and p-CHK2 protein levels. DNR, daunorubicin; p, phosphorylated.

In order to investigate the in vivo biocompatibility of this combinatorial therapy, tumor progression was investigated in a B-NSG MV4-11-luc xenograft mouse model by detecting fluorescence intensity. In addition, the $\mathrm{hCD} 45^{+}$cells in the bone marrow were quantified following lower extremity paralysis and the survival time of mice was then recorded. These in vivo experiments indicated that NL-101 in combination with DNR could significantly delay AML progression and prolong survival time, which supported the aforementioned in vitro results. The present findings further confirmed the efficacy and safety of this combinatorial therapy and may facilitate the future development of novel treatments.

The present study had certain limitations. Although the synergistic effects of the combination of NL-101 and DNR were examined and the underlying mechanisms were investigated, the most effective relative concentration between the two agents was not identified. With regards to in vivo experiments, the measurements of complete blood count and bone marrow smear could provide more information; therefore, a more comprehensive study is required. As DNR and Ara-C/idarubicin and Ara-C (DA/IA) regimen is the standard therapy for $\mathrm{AML}$, it might be better to compare the combination regimen with the DA/IA regimen in vivo. Moreover, the present study is a preliminary investigation that may facilitate the development of novel strategies based on the combination of NL-101 with other chemotherapeutics to improve the prognosis of patients with AML. However, it is unclear whether a combination of two chemotherapeutics may lead to an improved prognosis. Therefore, future studies are required to test additional combinations and to confirm the effectiveness of NL-101 + DNR in treating AML.

In conclusion, the present results suggested that the combinatorial treatment of NL-101 with DNR was effective in treating AML, and the key mechanism underlying this combination therapy was also identified. The combination of the SAHA-bendamustine hybrid NL-101 with DNR may represent a promising therapeutic strategy to treat patients with AML, thus improving their prognosis. 


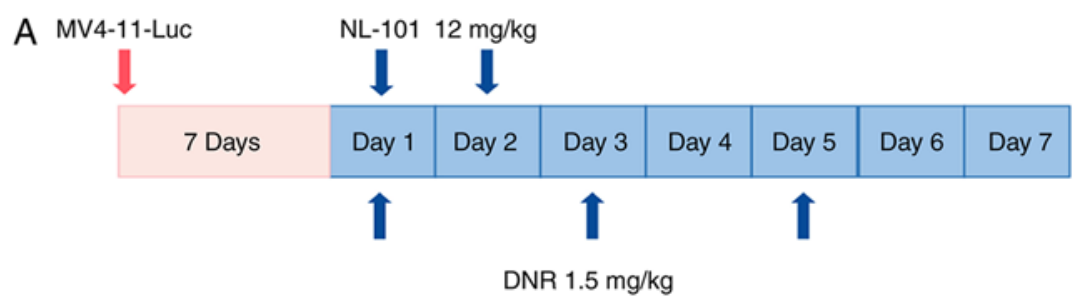

B Ctrl

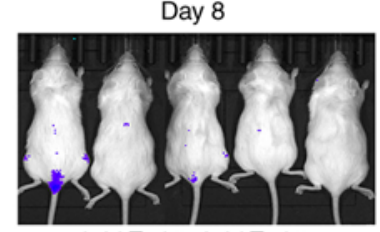

$9.82 \mathrm{E}+05 \pm 3.36 \mathrm{E}+05$

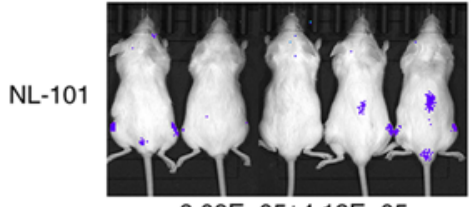

$8.09 E+05 \pm 4.13 E+05$

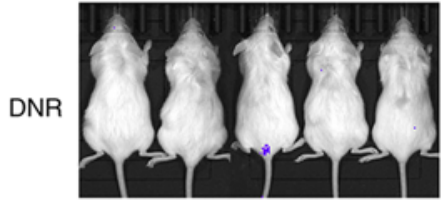

$5.22 \mathrm{E}+05 \pm 1.74 \mathrm{E}+05$

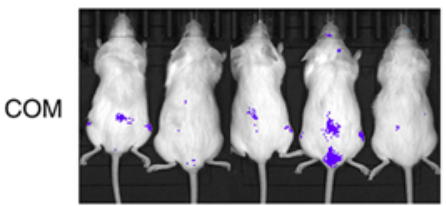

$9.24 \mathrm{E}+05 \pm 8.88 \mathrm{E}+04$

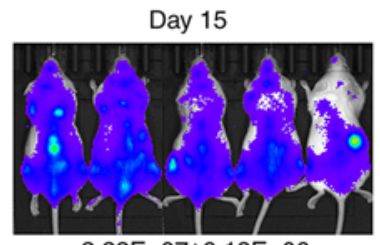

$2.22 \mathrm{E}+07 \pm 6.13 \mathrm{E}+06$

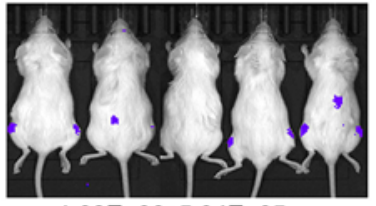

1.09E+06 $\pm 5.04 \mathrm{E}+05$

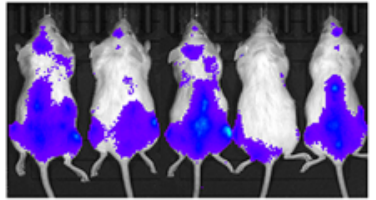

$9.62 \mathrm{E}+06 \pm 5.06 \mathrm{E}+06$

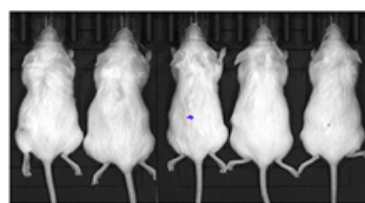

$2.61 \mathrm{E}+05 \pm 7.89 \mathrm{E}+04$

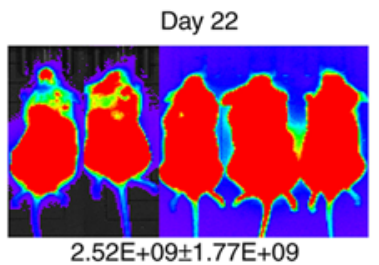

2.52E+09 $\pm 1.77 \mathrm{E}+09$

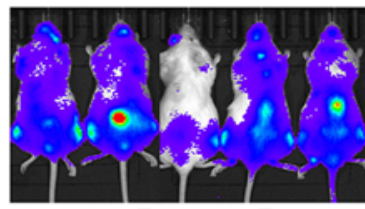

$2.98 \mathrm{E}+07 \pm 1.32 \mathrm{E}+07$

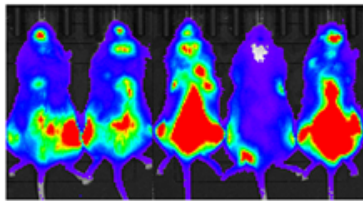

$2.59 E+08 \pm 1.62 E+08$

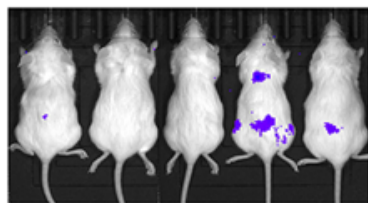

$1.10 \mathrm{E}+06 \pm 7.43 \mathrm{E}+05$

C

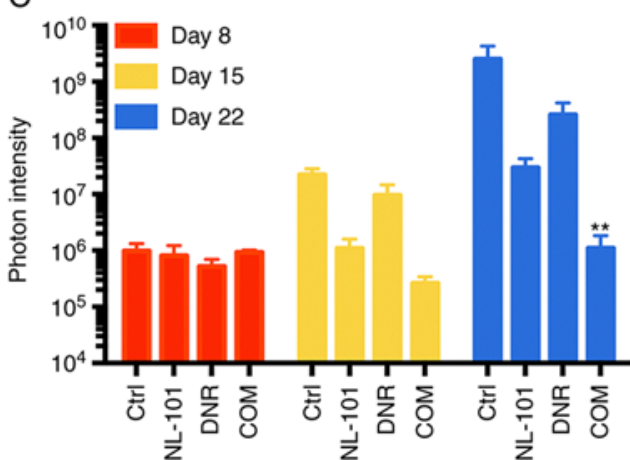

\author{
D
}
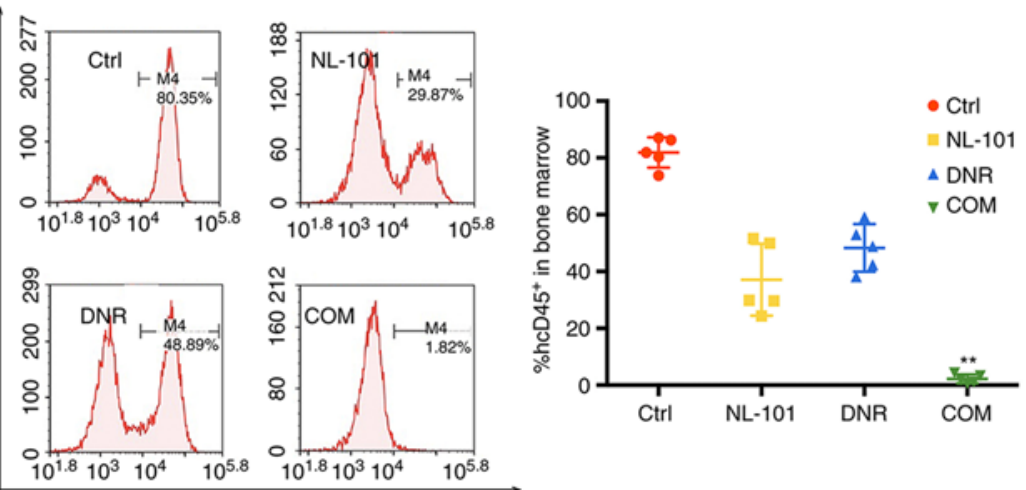

E

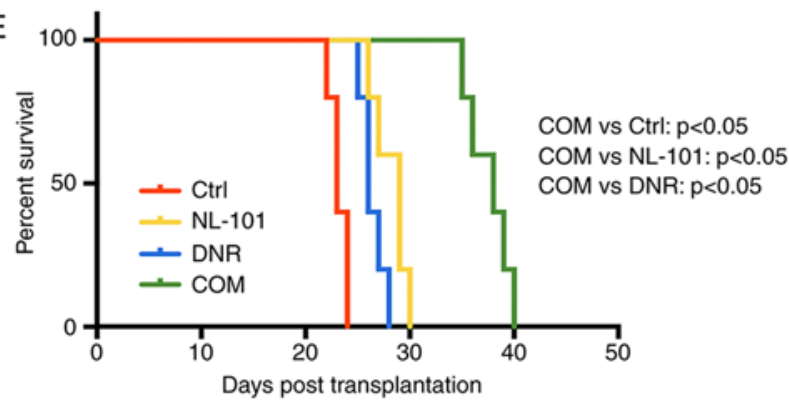

Figure 4. Anti-leukemic effect of NL-101, DNR and NL-101 + DNR on a murine model of leukemia. (A) Schematic diagram of the treatment regimen for the AML murine model. (B) Images and (C) measurements of photon intensity showing leukemia burden up to 22 days post-transplantation. (D) Representative flow cytometry histograms of hCD $45^{+}$blasts and a graph showing the percentage of hCD $45^{+}$blasts in the bone marrow of mice from different treatment groups. (E) Percentage survival. Data are presented as the mean \pm SD. P-values were determined using one-way ANOVA. ${ }^{* *}$ P $<0.001$ vs. NL-101 and DNR groups. COM, combination; Ctrl, control; DNR, daunorubicin; hCD45, human CD45. 


\section{Acknowledgements}

The authors would like to thank Professor Ravi Bhatia (City of Hope National Medical Center, Duarte, CA, USA) and Professor Jianjun Chen (City of Hope, Gehr Family Center for Leukemia Research, Duarte, CA, USA) for providing cell lines; Professor Rongzhen Xu (The Second Affiliated Hospital of Zhejiang University, Hangzhou, China) for supplying the MV4-11-luciferase cells, and Hangzhou Minsheng Institute of Pharmaceutical Research for providing NL-101.

\section{Funding}

This study was supported by Zhejiang Provincial Key Innovation Team (grant no.2011R50015) andZhejiang Provincial Natural Science Foundation of China (grant no. LY16H080002). The funders were not involved in experimental design, data analysis, manuscript writing and submission.

\section{Availability of data and materials}

All data generated or analyzed during this study are included in this published article.

\section{Authors' contributions}

JJ designed the experiments. JRJ, XL and WJG performed the experiments, and JRJ was a major contributor in writing the manuscript. FLL, JSH and XH analyzed the data. JJP and SJH collected the patient samples and information. WLY and QL contributed to the writing of the manuscript and performed the primary cells experiment. All authors read and approved the final manuscript.

\section{Ethics approval and consent to participate}

Animal experiments were approved by Animal Experimental Ethical Inspection of the First Affiliated Hospital, College of Medicine, Zhejiang University. All procedures performed involving human patients were approved by Research Ethics Committee of the First Affiliated Hospital, College of Medicine, Zhejiang University. Informed consent was obtained from all patients according to institutional guidelines.

\section{Patient consent for publication}

Not applicable.

\section{Competing interests}

The authors declare that they have no competing interests.

\section{References}

1. Lam SS, Ho ES, He BL, Wong WW, Cher CY, Ng NK, Man CH, Gill H, Cheung AM, Ip HW, et al: Homoharringtonine (omacetaxine mepesuccinate) as an adjunct for FLT3-ITD acute myeloid leukemia. Sci Transl Med 8: 359ra129, 2016.

2. Miller KD, Siegel RL, Lin CC, Mariotto AB, Kramer JL, Rowland JH, Stein KD, Alteri R and Jemal A: Cancer treatment and survivorship statistics, 2016. CA Cancer J Clin 66: 271-289, 2016.
3. Ferrara $\mathrm{F}$ and Schiffer CA: Acute myeloid leukaemia in adults. Lancet 381: 484-495, 2013.

4. Quintás-Cardama A, Santos FP and Garcia-Manero G: Histone deacetylase inhibitors for the treatment of myelodysplastic syndrome and acute myeloid leukemia. Leukemia 25: 226-235, 2011.

5. Papaemmanuil E, Gerstung M, Bullinger L, Gaidzik VI, Paschka P, Roberts ND, Potter NE, Heuser M, Thol F, Bolli N, et al: Genomic classification and prognosis in acute myeloid leukemia. N Engl J Med 374: 2209-2221, 2016.

6. Rosenblat TL, McDevitt MR, Mulford DA, Pandit-Taskar N, Divgi CR, Panageas KS, Heaney ML, Chanel S, Morgenstern A, Sgouros G, et al: Sequential cytarabine and alpha-particle immunotherapy with bismuth-213-lintuzumab (HuM195) for acute myeloid leukemia. Clin Cancer Res 16: 5303-5311, 2010.

7. Stein EM, DiNardo CD, Pollyea DA, Fathi AT, Roboz GJ, Altman JK, Stone RM, DeAngelo DJ, Levine RL, Flinn IW, et al: Enasidenib in mutant IDH2 relapsed or refractory acute myeloid leukemia. Blood 130: 722-731, 2017.

8. Döhner H, Weisdorf DJ and Bloomfield CD: Acute myeloid leukemia. N Engl J Med 373: 1136-1152, 2015.

9. Walter RB and Estey EH: Management of older or unfit patients with acute myeloid leukemia. Leukemia 29: 770-775, 2015.

10. Chen W, Zheng R, Baade PD, Zhang S, Zeng H, Bray F, Jemal A, Yu XQ and He J: Cancer statistics in China, 2015. CA Cancer J Clin 66: 115-132, 2016.

11. Löwenberg B and Rowe JM: Introduction to the review series on advances in acute myeloid leukemia (AML). Blood 127: 1, 2016.

12. Döhner H, Estey EH, Amadori S, Appelbaum FR, Büchner T, Burnett AK, Dombret H, Fenaux P, Grimwade D, Larson RA, et al: Diagnosis and management of acute myeloid leukemia in adults: Recommendations from an international expert panel, on behalf of the European LeukemiaNet. Blood 115: 453-474, 2010.

13. Wei AH and Tiong IS: Midostaurin, enasidenib, CPX-351, gemtuzumab ozogamicin, and venetoclax bring new hope to AML. Blood 130: 2469-2474, 2017.

14. Burnett A, Wetzler M and Löwenberg B: Therapeutic advances in acute myeloid leukemia. J Clin Oncol 29: 487-494, 2011.

15. Feldman EJ, Lancet JE, Kolitz JE, Ritchie EK, Roboz GJ, List AF, Allen SL, Asatiani E, Mayer LD, Swenson C and Louie AC: First-in-man study of CPX-351: A liposomal carrier containing cytarabine and daunorubicin in a fixed 5:1 molar ratio for the treatment of relapsed and refractory acute myeloid leukemia. J Clin Oncol 29: 979-985, 2011.

16. Lancet JE, Cortes JE, Hogge DE, Tallman MS, Kovacsovics TJ, Damon LE, Komrokji R, Solomon SR, Kolitz JE, Cooper M, et al: Phase 2 trial of CPX-351, a fixed 5:1 molar ratio of cytarabine/daunorubicin, vs. cytarabine/daunorubicin in older adults with untreated AML. Blood 123: 3239-3246, 2014.

17. Stein EM and Tallman MS: Emerging therapeutic drugs for AML. Blood 127: 71-78, 2016.

18. Besse L, Kraus M, Besse A, Bader J, Silzle T, Mehrling T and Driessen C: The first-in-class alkylating HDAC inhibitor EDO-S101 is highly synergistic with proteasome inhibition against multiple myeloma through activation of multiple pathways. Blood Cancer J 7: e589, 2017.

19. Mehrling T and Chen Y: The alkylating-HDAC inhibition fusion principle: Taking chemotherapy to the next level with the first in class molecule EDO-S101. Anticancer Agents Med Chem 16: 20-28, 2016.

20. Yu J, Qiu S, Ge Q, Wang Y, Wei H, Guo D, Chen S, Liu S, Li S, Xing $\mathrm{H}$, et al: A novel SAHA-bendamustine hybrid induces apoptosis of leukemia cells. Oncotarget 6: 20121-20131, 2015

21. Minotti G, Menna P, Salvatorelli E, Cairo G and Gianni L: Anthracyclines: Molecular advances and pharmacologic developments in antitumor activity and cardiotoxicity. Pharmacol Rev 56: 185-229, 2004.

22. Nishida Y, Mizutani N, Inoue M, Omori Y, Tamiya-Koizumi K, Takagi A, Kojima T, Suzuki M, Nozawa Y, Minami Y, et al: Phosphorylated Sp1 is the regulator of DNA-PKcs and DNA ligase IV transcription of daunorubicin-resistant leukemia cell lines. Biochim Biophys Acta 1839: 265-274, 2014.

23. Burden DA and Osheroff N: Mechanism of action of eukaryotic topoisomerase II and drugs targeted to the enzyme. Biochim Biophys Acta 1400: 139-154, 1998.

24. Murphy T and Yee KWL: Cytarabine and daunorubicin for the treatment of acute myeloid leukemia. Expert Opin Pharmacother 18: 1765-1780, 2017.

25. Drexler HG: Classification of acute myeloid leukemias-a comparison of FAB and immunophenotyping. Leukemia 1: 697-705, 1987. 
26. Zhao F, Sun X, Lu W, Xu L, Shi J, Yang S, Zhou M, Su F, Lin F and Cao F: Synthesis of novel, DNA binding heterocyclic dehydroabietylamine derivatives as potential antiproliferative and apoptosis-inducing agents. Drug Deliv 27: 216-227, 2020.

27. Zoi I, Karamouzis MV, Xingi E, Sarantis P, Thomaidou D, Lembessis P, Theocharis S and Papavassiliou AG: Combining RANK/RANKL and ERBB-2 targeting as a novel strategy in ERBB-2-positive breast carcinomas. Breast Cancer Res 21: 132, 2019.

28. Li X, Li C, Jin J, Wang J, Huang J, Ma Z, Huang X, He X, Zhou Y, $\mathrm{Xu}$ Y, et al: High PARP-1 expression predicts poor survival in acute myeloid leukemia and PARP-1 inhibitor and SAHA-bendamustine hybrid inhibitor combination treatment synergistically enhances anti-tumor effects. EBioMedicine 38: 47-56, 2018.

29. Tewari M, Quan LT, O'Rourke K, Desnoyers S, Zeng Z, Beidler DR, Poirier GG, Salvesen GS and Dixit VM: Yama/CPP32 beta, a mammalian homolog of CED-3, is a CrmA-inhibitable protease that cleaves the death substrate poly(ADP-ribose) polymerase. Cell 81: 801-809, 1995.

30. Kaufmann SH, Desnoyers S, Ottaviano Y, Davidson NE and Poirier GG: Specific proteolytic cleavage of poly(ADP-ribose) polymerase: An early marker of chemotherapy-induced apoptosis. Cancer Res 53: 3976-3985, 1993.

31. Testa U and Riccioni R: Deregulation of apoptosis in acute myeloid leukemia. Haematologica 92: 81-94, 2007.

32. Benito A, Silva M, Grillot D, Nuñez G and Fernández-Luna JL: Apoptosis induced by erythroid differentiation of human leukemia cell lines is inhibited by Bcl-XL. Blood 87: 3837-3843, 1996.
33. Nuñez G and Clarke MF: The Bcl-2 family of proteins: Regulators of cell death and survival. Trends Cell Biol 4: 399-403, 1994.

34. Cheson BD and Rummel MJ: Bendamustine: Rebirth of an old drug. J Clin Oncol 27: 1492-1501, 2009.

35. Garnock-Jones KP: Bendamustine: A review of its use in the management of indolent non-Hodgkin's lymphoma and mantle cell lymphoma. Drugs 70: 1703-1718, 2010.

36. Pang B, de Jong J, Qiao X, Wessels LF and Neefjes J: Chemical profiling of the genome with anti-cancer drugs defines target specificities. Nat Chem Biol 11: 472-480, 2015.

37. Pang B, Qiao X, Janssen L, Velds A, Groothuis T, Kerkhoven R, Nieuwland M, Ovaa $\mathrm{H}$, Rottenberg $\mathrm{S}$, van Tellingen $\mathrm{O}$, et al: Drug-induced histone eviction from open chromatin contributes to the chemotherapeutic effects of doxorubicin. Nat Commun 4: 1908, 2013

38. Rogakou EP, Pilch DR, Orr AH, Ivanova VS and Bonner WM: DNA double-stranded breaks induce histone H2AX phosphorylation on serine 139. J Biol Chem 273: 5858-5868, 1998.

39. Pilch DR, Sedelnikova OA, Redon C, Celeste A, Nussenzweig A and Bonner WM: Characteristics of gamma-H2AX foci at DNA double-strand breaks sites. Biochem Cell Biol 81: 123-129, 2003.

40. Zou L and Elledge SJ: Sensing DNA damage through ATRIP recognition of RPA-ssDNA complexes. Science 300: 1542-1548, 2003.

41. Reilly NM, Novara L, Di Nicolantonio F and Bardelli A: Exploiting DNA repair defects in colorectal cancer. Mol Oncol 13: 681-700, 2019.

42. Chen Y and Poon RY: The multiple checkpoint functions of CHK1 and CHK2 in maintenance of genome stability. Front Biosci 13: 5016-5029, 2008. 